\title{
Factors Influencing the Level of Oxygen Free Radicals in Female Nursing Students
}

\author{
Eun-Kyung $\mathrm{Kim}^{1} \&$ Mi Ryeong Song ${ }^{2}$ \\ ${ }^{1}$ Department of Nursing, Suwon Science College, Hwaseong, South Korea \\ ${ }^{2}$ College of Nursing, Gachon University, Incheon, South Korea \\ Correspondence: Mi Ryeong Song, College of Nursing, Gachon University, 191 Hambakmoe-ro, Younsu-gu, \\ Incheon, South Korea. Tel: 82-102-038-2384. E-mail: miryeong@gachon.ac.kr
}

\author{
Received: April 21, 2019 Accepted: May 12, 2019 Online Published: May 16, 2019 \\ doi:10.5539/gjhs.v11n7p1 URL: https://doi.org/10.5539/gjhs.v11n7p1
}

\begin{abstract}
This study was conducted to investigate the levels of oxygen free radicals and related health factors in 201 female nursing students. The questionnaire was completed by the participants and their oxygen free radical levels were measured by urine test. In this study, an oxygen free radical analyzer was used to measure oxygen free radical levels. The oxygen free radical analyzer analyzes the amount of oxygen free radicals in the body by measuring urinary malondialdehyde (MDA). To determine factors associated with oxygen free radical levels, multiple regression tests were conducted. Of the participants, $89.6 \%$ exhibited normal levels of oxygen free radicals and $10.4 \%$ had elevated levels. In this study, the factors that affected oxygen free radical levels were eating habit $(\beta$ $=.20, p=.003)$, fatigue $(\beta=.20, p=.004)$, and detox necessity $(\beta=.18, p=.006)$. In order to lower oxygen free radical levels of female nursing students, the areas of eating habit, fatigue, and detox must receive increased focus.
\end{abstract}

Keywords: students, oxygen free radical, eating habit, fatigue, detox

\section{Introduction}

\subsection{Necessity of Research}

As modern health risk factors such as environmental pollution, lack of exercise, misinformed dieting, unbalanced dietary intake, and stress have increased, the disease pattern has also changed. Oxidative stress plays an important role in the occurrence and persistence of inflammation, and can eventually cause cardiovascular disease, diabetes, cancer, or neural degeneration (Lugrin, Rosenblatt-Velin, Parapanov, \& Liaudet, 2014). Oxidative stress occurs when there is an imbalance between oxygen free radical production and antioxidants (Valko, Rhodes, Moncol, Izakovic, \& Mazur, 2006). There is increasing scientific interest in oxygen free radicals, which are the main cause of oxidative stress.

"Oxygen free radical" is a generic term for various oxygen-containing compounds that are unstable and exhibit increased reactivity. Oxygen free radicals are generated during the breathing process and in other life-sustaining activities of human beings. In cytoplasmic mitochondria, nutrients and oxygen are combined and converted into energy. In this state, electrons are attached, and the highly reactive compound has a short life span. If oxygen free radicals are properly available, the human body will use them to kill germs and cancer cells. However, an excess of oxygen free radicals in the body can lead to oxidative stress, which can cause aging or disease from DNA, RNA, enzymes, or cell membrane damage (Kang, 2012; Mastaloudis et al., 2004). Oxygen free radicals can be generated as long as humans breathe and perform life-sustaining activities. Of the oxygen entering the body through respiration, $2-5 \%$ is converted to oxygen free radicals; there are four main types of oxygen free radicals: the superoxide anion $\left(\mathrm{O}_{2}-\right)$, hydrogen peroxide $\left(\mathrm{H}_{2} \mathrm{O}_{2}\right)$, the hydroxyl radical $\left(\mathrm{OH}^{-}\right)$, and singlet oxygen $\left({ }^{1} \mathrm{O}_{2}\right)(\mathrm{Kang}$, 2012). The human body responds by generating antioxidants (alpha-tocopherol, beta-carotene, catalase, superoxide dismutase (SOD), glutathione, etc.) to defend against oxygen free radicals. However, when the level of oxygen free radicals generated exceeds the amount tolerated by the antioxidants, oxidative stress occurs.

Excessive production of oxygen free radicals is affected by many factors, including obesity; lack of exercise; excessive exercise; poor eating habits, such as overeating or frequent ingestion of instant food; radiation and environmental pollution; fatigue and overwork; and drinking and smoking (Choi \& Kim, 2014; Fogarty et al., 2011). According to the Korean National Health and Nutrition Examination Survey (2017), 37.3\% of male in the 
19-29 age group smoked, compared with $9.7 \%$ in the same age female group (Korea Statistical Information Service, 2017). The difference in smoking rates between men and women was thought to affect the level of free radicals. Female nursing students are included in this study because most nursing students are female students.

This study investigated oxygen free radical level as an index of healthcare in female nursing students who will become nursing specialists. We determined the effects of obesity (Body Mass Index: BMI), exercise, eating habit, exposure to environmental pollution, fatigue, health perception, and detox (detoxification) necessity on oxygen free radical levels. This will be the basis for proper health management education to reduce oxygen free radical levels in female nursing students.

\subsection{Research Objectives}

1) To identify the level of oxygen free radicals and analyze the differences in oxygen free radical levels according to socio-demographic characteristics of female nursing students.

2) To identify health-related characteristics of female nursing students.

3) To investigate the correlation between female nursing students' health-related characteristics and oxygen free radical levels.

4) To identify the influencing factors on elevated oxygen free radical levels in female nursing students.

\section{Methods}

\subsection{Research Design}

This study is a descriptive study to identify female nursing students' oxygen free radical levels and correlated factors.

\subsection{Research Participants}

After obtaining permission to collect data from a nursing college, we recruited participants through the bulletin board. We explained to the nursing college students who wanted to participate in the study to measure free radicals using urine and collect data through questionnaires. The data were collected from 201 students who signed the consent form. Male nursing students, considered a minority, were excluded due to possible physiological sex-based differences. In addition, junior and senior students who had experience of hospital practice were included. The minimum required sample size was estimated using the $\mathrm{G} *$ power program (Faul, Erdfelder, Buchner, \& Lang, 2009). On the basis of multiple regression, 174 subjects were required for significance level of .05, power of 0.95 , effect size (f2) of 0.11 , and number of predictors of 4 . Data were collected from 201 students in consideration of dropout rate, and there were no dropouts.

\subsection{Research Tools}

\subsubsection{Oxygen Free Radical Levels}

An oxygen free radical is a highly reactive atom or group of atoms containing an unpaired electron. A free radical containing oxygen can be created in the body during the breathing process. It is then oxidized by attacking biological tissues and damaging cells that are generated during metabolic processes (Lugrin et al., 2014). In this study, an oxygen free radical analyzer (BS201 model, BioNics) was used to measure oxygen free radical levels. The BS201 oxygen free radical analyzer analyzes the amount of oxygen free radicals in the body by measuring urinary malondialdehyde (MDA). MDA is a small molecular aldehyde consisting of three carbons. It is a decomposition product of peroxide and is generated by the attack of a oxygen free radical on a polyunsaturated fatty acid. It is known that fat peroxidation is caused by highly reactive oxygen molecules, especially oxygen free radicals. Thus, MDA is an accurate measurement to determine oxygen free radical content in the body (Mukhopadhyay, Gongopadhyyay, Rani, Gaver, \& Mishra, 2015; Ozmen et al., 2009).

Before oxygen free radical levels were measured, the BS201 was connected to the computer and the program to perform the calibration was activated. For the test procedure, the urine was stripped and placed in the analyzer. After 3 minutes, the amount of oxygen free radicals was measured. The criteria were: $0-500 \mathrm{fp}$ (free radical point), normal; 501-1,000 fp, needs attention; 1,001-3,000 fp, somewhat high; 3,001-5,000 fp, high; and 5,001 fp and above, very high.

\subsubsection{Body Mass Index (BMI)}

BMI is used to indirectly measure obesity using body mass and height measurements. BMI is calculated as mass divided by the square of height. The units are $\mathrm{kg} / \mathrm{m}^{2}$. By the Korean Society of Obesity's standards, a BMI under 18.4 is classified as underweight, 18.5-22.9 is normal, 23.0-24.9 is overweight, and over 25.0 is obese. 


\subsubsection{Exercise}

Participants were asked, "How often do you exercise?" Their answers were based on a five points scale ranging from 1 point ("not at all") to 5 points ("very frequently"), meaning that the higher the score, the more frequently the participant exercises.

\subsubsection{Eating Habit}

To measure usual eating habits, each participant was given five diet-related statements. The statements were "I often overeat," "I often eat midnight snack," "I often eat instant food (ramen, hamburger, etc.)," "I often eat fried food," and "I often eat synthetically-seasoned foods." For each item, participants gave a point value ranging from one ("not at all") to five ("very much"). The mean scores of the five items were calculated. The higher the score, the worse the eating habit is. Cronbach's $\alpha$ was 0.60 in this study.

\subsubsection{Exposure to Exhaust Fumes and Dust}

To measure the degree of exposure to environmental pollution, we gave the participants two statements: "Usually, I am heavily exposed to exhaust fumes" and "Usually, I am heavily exposed to dust." For each item, participants reported a point value ranging from one ("not at all") to five ("very much"). The mean scores of the two items were calculated. The higher the score, the greater the exposure to exhaust fumes and dust.

\subsubsection{Fatigue}

The participants were asked, "What is your usual fatigue level, assuming that 100 points correspond to the maximum fatigue?" The participants gave direct point values from 0 (minimum fatigue) to 100 (maximum fatigue), meaning that the higher the score, the higher the fatigue.

\subsubsection{Awareness of Health}

We asked, "How do you think your health condition is?" Participants gave points ranging from one ("very bad") to five ("very good"), meaning that the higher the score, the better they perceived their health.

\subsubsection{Detox Necessity}

We asked, "How much do you think a detox is necessary for you?" We defined "detox" as "to release toxic substances and wastes accumulated in the body." Participants gave points ranging from one ("not necessary at all") to five ("very necessary"), meaning that the higher the score, the more necessary the detox.

\subsection{Data Collection and Analysis Methods}

The study was conducted from April to August 2016 on female nursing students from a university in a region. The purpose and method of the study were explained to them and consent forms were received from all participants. The questionnaire was completed by the participants and their oxygen free radical levels were measured by urine test, which took 15-20 minutes. The meaning of their oxygen free radical results, the effects of oxygen free radicals on the human body, and methods for reducing oxygen free radicals were briefly explained to the participants.

For data analysis, this study used descriptive statistics for demographic characteristics and health-related characteristics and analyzed oxygen free radical levels differences according to demographic characteristics with the independent $t$-test or ANOVA statistical test. Correlations between health-related characteristics and oxygen free radical levels were analyzed using Pearson's correlation tests. To determine factors associated with oxygen free radical levels, multiple regression tests were conducted.

\subsection{Ethical Considerations}

Prior to the start of this research, the author submitted a research proposal to the Institutional Review Board of the affiliated institution. To comply with ethical considerations, the researcher explained the goals and the content of the study to the participants, as well as their right to refuse to participate at any stage, how their personal information would be protected, and what compensation they would receive for their participation.

\section{Results}

\subsection{Difference in Oxygen Free Radical Levels According to Socio-Demographic Characteristics}

For this study, $51.7 \%$ of the participants were seniors and $48.3 \%$ were juniors. Additionally, $58.7 \%$ were non-religious, and more than half (67.7\%) had middle-class economic status. The mean oxygen free radical level of the participants was $200.41 \mathrm{fp}$, the maximum was $562.00 \mathrm{fp}$, and the minimum was $122.00 \mathrm{fp}$. Of the participants, $89.6 \%$ had normal levels (less than $500 \mathrm{fp}$ ), and 10.4\% had elevated levels of 501-1,000 fp. There were no differences in oxygen free radical levels according to socio-demographic characteristics (Table 1). 
Table 1. Differences in oxygen free radical levels according to socio-demographic characteristics $(n=201)$

\begin{tabular}{|c|c|c|c|c|c|c|}
\hline \multirow{2}{*}{ Characteristics } & \multirow{2}{*}{ Category } & \multirow{2}{*}{$\begin{array}{l}\text { Total } \\
\text { N (\%) }\end{array}$} & \multicolumn{3}{|c|}{ Oxygen free radical } & \multirow{2}{*}{ Minimum-Maximum } \\
\hline & & & Mean (SD) & $\mathrm{t} / \mathrm{F}$ & $p$ & \\
\hline \multirow{2}{*}{ Oxygen free radical (fp) } & $<500$ (normal) & $180(89.6)$ & $200.41(129.81)$ & \multirow{2}{*}{ - } & \multirow{2}{*}{-} & \multirow{2}{*}{$122.00-562.00$} \\
\hline & $501-1,000$ (elevated) & $21(10.4)$ & & & & \\
\hline \multirow{2}{*}{ Year } & Junior & $97(48.3)$ & $254.61(168.18)$ & \multirow{2}{*}{6.23} & \multirow{2}{*}{.067} & \\
\hline & Senior & $104(51.7)$ & $219.86(131.83)$ & & & \\
\hline \multirow{2}{*}{ Religious } & Yes & $83(41.3)$ & $225.28(152.19)$ & \multirow{2}{*}{2.30} & \multirow{2}{*}{.058} & \\
\hline & No & $118(58.7)$ & $200.92(108.75)$ & & & \\
\hline \multirow{3}{*}{ Economic status } & Upper-class & $24(11.9)$ & $177.33(88.89)$ & \multirow{3}{*}{4.04} & \multirow{3}{*}{.218} & \\
\hline & Middle-class & $136(67.7)$ & $189.38(114.51)$ & & & \\
\hline & Lower-class & $41(20.4)$ & $180.51(179.31)$ & & & \\
\hline
\end{tabular}

\subsection{Health-Related Characteristics of Female Nursing Students}

The mean BMI of the participants was 20.78. Of the participants, $19.9 \%$ were underweight, $61.7 \%$ had a normal weight, $11.4 \%$ were overweight, and $7.0 \%$ were obese (according to the Korean Society of Obesity's criteria). The mean usual exercise level was 2.85 points, which was between "almost not frequent" and "normal." The mean eating habit was 3.45 points, which was between "mostly eat often" and "normal" for levels of overeating, instant food, midnight snacks, fried food intake, and synthetic seasoning intake. The mean level of exposure to environmental pollution was 2.95 points, which was between "almost not exposed" and "normal". The mean level of fatigue was 68.72 points (on a scale of $0-100$ points). The mean health awareness was 3.25 points, meaning that the health of the participants was self-perceived as between "normal" and "generally good." The mean level of need for a detox was 3.69 points, which was considered to be between "normal" and "almost necessary" (Table 2).

Table 2. Health-related characteristics of the participants $(n=201)$

\begin{tabular}{lccc}
\hline Health-related characteristics & Category & $\mathrm{N}(\%)$ & Mean (SD) \\
\hline & $\leq 18.4$ & $40(19.9)$ & \\
Body mass index & $18.5-22.9$ & $124(61.7)$ & $20.78(2.69)$ \\
& $23.0-24.9$ & $23(11.4)$ & \\
\hline Exercise & $25.0 \leq$ & $14(7.0)$ & $2.85(1.07)$ \\
Eating habits & & $3.45(.63)$ \\
Exposure to pollution & & $2.95(.96)$ \\
Fatigue & & $68.72(21.11)$ \\
Health awareness & & $3.25(.86)$ \\
Detox necessity & & $3.69(.90)$ \\
\hline
\end{tabular}

\subsection{Correlation Between Female Nursing Students' Health-Related Characteristics and Oxygen Free Radical Levels}

Upon examining the health-related characteristics and oxygen free radical levels in study participants, a significant negative correlation between BMI and oxygen free radical level $(\mathrm{r}=-0.14, p=0.038)$ was found. The oxygen free radical level had a significant positive correlation with unhealthy eating habit $(\mathrm{r}=0.18, p=0.009)$, fatigue $(\mathrm{r}=$ $0.17, p=0.014)$, and awareness of detox necessity $(\mathrm{r}=0.16, p=0.018)$. When levels of unhealthy eating habits, fatigue, and need to detox were higher, oxygen free radical levels were significantly higher as well (Table 3 ). 
Table 3. Correlation between health-related characteristics and oxygen free radical levels

\begin{tabular}{llllllll}
\hline & 1 & 2 & 3 & 4 & 5 & 6 & 7 \\
\cline { 2 - 7 } & $\mathrm{r}(p)$ & & & & & & \\
\hline 1. Oxygen free radical & 1 & & & & & & \\
2. Body mass index & $-.14(.038)$ & 1 & & & & & \\
3. Exercise & $-.03(.606)$ & $.07(.283)$ & 1 & & & \\
4. Eating habits & $.18(.009)$ & $.06(.332)$ & $-.14(.034)$ & 1 & & \\
5. Exposure to pollution & $.05(.445)$ & $.05(.461)$ & $-.06(.335)$ & $.18(.010)$ & 1 & \\
6. Fatigue & $.17(.014)$ & $.02(.682)$ & $-.19(.005)$ & $.07(.324)$ & $.14(.040)$ & 1 & \\
7. Health awareness & $-.07(.279)$ & $-.08(.218)$ & $.16(.016)$ & $.00(.902)$ & $-.17(.012)$ & $-.31(<.001)$ & 1 \\
8. Detox necessity & $.16(.018)$ & $.26(<.001)$ & $.04(.560)$ & $-.02(.708)$ & $.05(.482)$ & $.07(.323)$ & $-.041(.528)$ \\
\hline
\end{tabular}

\subsection{Influencing factors of oxygen free radical levels in female nursing students}

To further investigate the variables affecting the oxygen free radical levels of the study participants, multiple regression analysis using the stepwise input method was conducted for the variables, which showed a significant correlation with oxygen free radical levels (BMI, eating habit, fatigue, and detox necessity). When there were worse eating habit $(\beta=.20, p=.003)$, higher fatigue $(\beta=0.20, p=0.004)$, and more detox necessity $(\beta=0.18, p=$ $0.006)$, the oxygen free radical level was significantly higher. The explanatory power of these variables was $10.3 \%$ $\left(\mathrm{R}^{2}=0.103, p<0.001\right)($ Table 4$)$.

Table 4. Influencing variables of oxygen free radical levels $(n=201)$

\begin{tabular}{lrrrrrrr}
\hline & $\mathrm{B}$ & $\mathrm{S} . \mathrm{E}$. & $\beta$ & $\mathrm{R}^{2}$ & $\mathrm{t}$ & $p$ & $\mathrm{~F}(p)$ \\
\hline Eating habits & 41.07 & 13.79 & .20 & .033 & 2.97 & .003 & $7.51(<.001)$ \\
Fatigue & 1.23 & .41 & .20 & .068 & 2.95 & .004 & \\
Detox necessity & 26.83 & 9.73 & .18 & .103 & 2.75 & .006 & \\
\hline
\end{tabular}

\section{Discussion}

This study was conducted to investigate oxygen free radical levels and possibly related variables in female nursing students. Of the participants, $89.6 \%$ had normal levels of oxygen free radicals and the remaining $10.4 \%$ had elevated levels. In a study of oxygen free radical levels in female college students similarly aged to the participants in this study (Choi \& Kim, 2014), 41.8\% of the participants had "normal" levels. Of those with "abnormal" levels, $36.3 \%$ were "caution," $9.1 \%$ had "low oxidative stress," $1.8 \%$ had "medium oxidative stress," and $3.6 \%$ had "high oxidative stress". In a study (Kang, 2012) that measured the oxygen free radical levels in adult males, only $7.4 \%$ of the participants were "normal." Of those at the "abnormal" level, 36.4\% were "caution." $19.5 \%$ had "low oxidative stress," $22.5 \%$ had "medium oxidative stress," and $14.1 \%$ had "high oxidative stress." Both of these previous studies have fewer participants with "normal" levels of oxygen free radicals. In addition to having more "abnormal" participants, these studies also had participants in the higher "oxidative stress" stages. In this study, the highest level of abnormality attained by participants was the "caution" state. This may be because female nursing students, the participants of this study, are relatively well able to reduce oxygen free radical production compared to other college students or similarly aged men.

On the other hand, with regard to oxygen free radical level according to sex, there are reports that oxygen free radical levels in females are significantly higher than those of males (Khadir et al., 2015), and that females are more vulnerable to oxidative stress (Topic et al., 2016). However, another report states that there are no differences between the sexes (Kirshbaum, 2002). In the present study, male nursing students, considered a minority, were excluded due to possible physiological sex-based differences. Therefore, it is necessary to further study oxygen free radical levels of female and male according to sex.

As for health-related characteristics affecting oxygen free radical levels, it was found that worse eating habits was significantly correlated with higher oxygen free radical levels. It is therefore necessary to correct bad eating habits 
such as overeating, ingesting instant foods, and frequently eating midnight snack, fried foods, and foods with synthetic seasonings. Conversely, regularity of meals, number of snacks consumed, and total mealtime are not significantly correlated with oxygen free radical levels (Choi \& Kim, 2014). Other studies found that oxygen free radical levels were significantly higher in groups that either did not eat fruit more than once a day (Lee et al., 2012) or had a high fat intake (Reddy, Ramamurthy, Somasekaraiah, Reddy, \& Rao, 1997) Therefore, it is more important to focus on avoiding overeating than on factors such as meal regularity or total mealtime. To reduce oxygen free radical levels, one must refrain from consuming foods containing fried foods, foods with synthetic seasonings, and instant foods, and must consume many fruits.

Another health-related characteristic that affects oxygen free radical levels is fatigue. It was found that higher fatigue was correlated with higher oxygen free radical level. In a study measuring oxidative stress in 56 patients with chronic fatigue syndrome (Maes, Kubera, Uytterhoeven, Vrydags, \& Bosmans, 2011), the patients with chronic fatigue syndrome were reported to have significantly higher oxidative stress than the control group, suggesting that there is an association between chronic fatigue and oxidative stress. In the present study, fatigue had a significant negative correlation with exercise level and health awareness. Lesser amounts of exercise were associated with higher levels of fatigue. Higher amounts of fatigue were associated with worse health awareness. Therefore, it can be assumed that lowering fatigue by practicing regular exercise can not only lower oxygen free radical level, but also increase awareness of health.

It is reported that when oxygen free radical levels exceed those able to be fought by the body's antioxidant supply, exercise may increase antioxidant capacity (Simioni et al., 2018). However, the relationship between exercise and oxygen free radical level is affected by exercise intensity. This is because excessive high-intensity exercise increases the oxygen demand in the body by 10-15 times, and oxygen free radical levels are increased by adenine nucleotide catabolism caused by skeletal muscle ischemia (Lamina, Ezema, Theresa, \& Anthonia, 2013; Vollaard, Shearman, \& Cooper, 2005). Therefore, in order to lower oxygen free radical levels, it is important to avoid strong and sudden movement and to exercise to a degree appropriate to one's physical strength (Mastaloudis et al., 2004). Oxygen free radical levels are also affected by exercise duration. One study measured oxygen free radical amounts in elderly females who conducted exercise regimens combining walking and Thera Band exercise for six or twelve weeks. It was reported that participants in the six-week exercise program had unchanged amounts of oxygen free radicals, but participants in the twelve-week exercise program had significantly decreased oxygen free radical levels. Therefore, it is seen that a long-term regular exercise program may lower oxygen free radical levels, while a short-term exercise program is ineffective (Alikani \& Sheikholeslami-Vatani, 2019).

Lastly, a health-related characteristic that is associated with oxygen free radical levels is awareness of detox necessity. It was found that participants that felt a higher need to detox had a higher oxygen free radical level. A high self-rated detox necessity may predict certain physiological situations. It is possible that one would be more likely to perceive the need for a detox in situations where he or she feels obese, does not exercise regularly, or feels tired at all times. Therefore, a high awareness of the need for detox would mean that there are unhealthy lifestyle habits that need to be corrected. Alternatively, a high self-rated detox necessity could mean that the subject is more likely to practice the detoxifying habits. However, in the present study, no significant correlations between detox necessity and the other health characteristics (exercise, eating) were found. These results suggest that even though someone is aware of the need to detox, they do not necessarily exercise or eat more healthily. In other words, they do not carry out activities that are associated with lower levels of oxygen free radicals. Therefore, it is necessary to provide strategies to induce healthier habits by providing concrete exercise methods or eating habit improvement measures. Then, female college students who are highly aware of their need to detox can engage in habits that are associated with lower oxygen free radical levels.

In conclusion, lowering oxygen free radical levels cannot be overlooked in healthcare. Of female nursing students, those who have bad eating habits, usually feel fatigued, or feel the need for a detox, are particularly at risk for high oxygen free radical levels. It is necessary to provide specific education to help these populations to lower oxygen free radical levels.

\section{Competing Interests Statement}

The authors declare no conflict of interest.

\section{References}

Alikhani, S., \& Sheikholeslami-Vatani, D. (2019). Oxidative stress and anti-oxidant responses to regular resistance training in young and older adult women. Geriatrics \& Gerontology International, 19(5), 419-422. https://doi.org/10.1111/ggi.13636 
Choi, S-N., \& Kim, H-J. (2014). Dietary behaviors and free radical level of smoking college women in Gyeong-gi province. The Korea Journal of Sports Science, 23(2), 1269-1282.

Faul, F. Erdfelder, E., Buchner, A., \& Lang, A. G. (2009). Statistical power analyses using G*Power 3.1: Tests for correlation and regression analyses. Behavior Research Methods, 41, 1149-1160. http://doi.org/10.3758/BRM.41.4.1149

Fogarty, M. C., Hughes, C. M., Burke, G., Brown, J. C., Trinick, T. R., Duly, E., ... Davison, G. W. (2011). Exercise-induced lipid peroxidation: implications for deoxyribonucleic acid damage and systemic free radical generation. Environmental and Molecular Mutagenesis, 52(1), 35-42. https://doi.org/10.1002/em.20572

Kang, Y. J. (2012). The correlation between the smoking and oxygen free radicals in men (Unpublished master's thesis). University of Ajou, Seoul, South Korea.

Khadir, A., Tiss, A., Kavalakatt, S., Behbehani, K., Dehbi, M., \& Elkum, N. (2015). Gender-specific association of oxidative stress and inflammation with cardiovascular risk factors in Arab population. Mediators of Inflammation, E-pub 2015 Mar 31. https://doi.org/10.1155/2015/512603

Kirschbaum, B. (2002). Correlative studies of urine fluorescence and free radical indicators. Clinical Nephrology, 58(5), 344-349.

Korea Statistical Information Service. (2017). Present smoking rate. Daejeon: Korea Statistical Information Service. Retrieved

from http://kosis.kr/statHtml/statHtml.do?orgId=117\&tblId=DT_11702_N001\&conn_path=I2

Lamina, S., Ezema, C. I., Theresa, A. I., \& Anthonia, E. U. (2013). Effects of free radicals and antioxidants on exercise performance. Oxidants and Antioxidants in Medical Science, 2(2), 83-91. http://doi.org/10.5455/oams.010413.rv.005

Lee, Y-A., Kang, S-G., Kim, S-H., Park, S-J., Kim, H-N., Song, I-S., ... Song, S-W. (2012). Assessment of lifestyle effects on the levels of free oxygen radicals in the Korean population. Korean Journal of Family Medicine, 33(5), 296-304. https://doi.org/10.4082/kjfm.2012.33.5.296

Lugrin, J., Rosenblatt-Velin, N., Parapanov, R., \& Liaudet, L. (2014). The role of oxidative stress during inflammatory processes. Biological Chemistry, 395(2), 203-230. https://doi.org/10.1515/hsz-2013-0241

Maes, M., Kubera, M., Uytterhoeven, M., Vrydags, N., \& Bosmans, E. (2011). Increased plasma peroxides as a marker of oxidative stress in myalgic encephalomyelitis/chronic fatigue syndrome (ME/CFS). Medical Science Monitor, 17(4), SC11-5. http://doi.org/10.12659/MSM.881699

Mastaloudis, A., Yu, T. W., O’Donnell, R. P., Frei, B., Dashwood, R. H., \& Traber, M. G. (2004). Endurance exercise results in DNA damage as detected by the comet assay. Free Radical Biology \& Medicine, 36(8), 966-975. https://doi.org/10.1016/j.freeradbiomed.2004.01.012

Mukhopadhyay, B., Gongopadhyay, A. N., Rani, A., Gaver, R., \& Mishra, S. P. (2015). Free radicals and antioxidants status in neonates with congenital malformation. Journal of Indian Association of Pediatric Surgeons, 20(4), 179-183. https://doi.org/10.4103/0971-9261.161037.

Ozmen, M. M., Zulfikaroglu, B., Col, C., Cinel, I., Isman, F. K., Cinel, L., ... Besler, T. H. (2009). Effect of increased abdominal pressure on cytokines (IL 1 beta, IL6, TNF alpha), C-reactive protein (CRP), free radicals (NO, MDA), and histology. Surgical Laparoscopy, Endoscopy \& Percutaneous Techniques, 19(2), 142-147. https://doi.org/10.1097/SLE.0b013e31819cdda7.

Reddy, K. K., Ramamurthy, R., Somasekaraiah, B. V., Reddy, T. P., \& Rao, P. (1997). Free radical and antioxidant status in urban and rural Tirupati men: interaction with nutrient intake, substance abuse, obesity and body fat distribution. Asia Pacific Journal of Clinical Nutrition, 6(4), 296-311.

Simioni, C., Zauli, G., Martelli, A., Vitale, M., Sacchetti, G., Gonelli, A., ... Neri, L. M. (2018). Oxidative stress: role of physical exercise and antioxidant nutraceuticals in adulthood and aging. Oncotarget, 9(24), 17181-17198. https://doi.org/10.18632/oncotarget.24729.

Topic, A., Malic, Z., Francuski, D., Stankovic, M., Markovic, B., Soskic, B., ... Radojkovic, D. (2016). Gender-related differences in susceptibility to oxidative stress in healthy middle-aged Serbian adults. Biomarkers, 21(2), 186-193. https://doi.org/10.3109/1354750X.2015.1126647

Valko, M., Rhodes, C. J., Moncol, J., Izakovic, M., \& Mazur, M. (2006). Free radicals, metals and antioxidants in 
oxidative stress-induced cancer. Chemico-Biological Interactions, 160(1), 1-40. https://doi.org/10.1016/j.cbi.2005.12.009

Vollaard, N. B., Shearman, J. P., \& Cooper, C. E. (2005). Exercise-induced oxidative stress. Myths, realities and $\begin{array}{lllll}\text { physiological } & \text { relevance, } & \text { Sports } & \text { Medicine, } & \text { 35(12), }\end{array}$ https://doi.org/10.2165/00007256-200535120-00004

\section{Copyrights}

Copyright for this article is retained by the author(s), with first publication rights granted to the journal.

This is an open-access article distributed under the terms and conditions of the Creative Commons Attribution license (http://creativecommons.org/licenses/by/4.0/). 